\title{
Ethnobotanical usage of plants as aphrodisiac agents in Anatolian folk medicine
}

\author{
Selin Tufan, Gizem Gülsoy Toplan *, Afife Mat \\ Department of Pharmacognosy, Faculty of Pharmacy, İstanbul University, 34116 İstanbul, Turkey \\ * Correspondence: eczgizemgulsoy@gmail.com, gizem.toplan@istanbul.edu.tr (G.G.T.); Tel: +90 21244000 00; \\ ORCID No: 0000-0002-0544-2532.
}

Submitted: 24 June 2017 / Revised: 08 August 2017 / Accepted: 14 August 2017

\begin{abstract}
Several plants have been used as aphrodisiac to treat sexual problems for centuries. Nowadays, despite the synthesis of chemicals, herbal medicines continue to maintain their importance. Turkey has a great tradition of folk medicine due to its rich flora and many plants have been used for the treatment of several disorders. This work presents aphrodisiac plants traditionally used in Turkey, with their local names, used parts, usage and administration route.

KEYWORDS: Aphrodisiac plants; folk medicine; ethnobotanical usage; aphrodisiacs
\end{abstract}

\section{INTRODUCTION}

The word aphrodisiac is originated from Aphrodite, The Greek Goddess of love and beauty [1]. With all respect to her duty she is responsible from the lovely ways of creating human relations. She is spreading love potions all over the humans and she has been decorating nature with colors with the help of trees and flowers. According to mythology, when Aphrodite born from seafoam and set foot on earth face, different types of flowers started to blossom under her foot and since that day, followers of Aphrodite have been able to use those flowers (which are called "Plants of Love") as aphrodisiac [2-4].

Since the beginning of humankind, sexuality has been really important in terms of continuation of the race and transfer of the culture to the offsprings. The usage of aphrodisiacs has been developed during centuries. Some of them provide psychological relief, while others affect physical system to treat sexual disorders [5-7].

Sexual relationship is very important in human life and it influences the social relationship between partners [1]. The cause of sexual disorders can be physical or psychological, and sometimes both. Today, both of them are lying under the majority of problems [8]. Nowadays, chronic disorders, antipsychotic and antidepressant therapeutic drugs, tobacco, stress, chronic alcohol abuse have increased these problems. The most common sexual problems in men are ejaculation disorders, erectile dysfunction, and loss of libido. In $25 \%$ to $63 \%$ of women, sexual dysfunction is also a serious problem [6-8].

By definition, aphrodisiacs are materials that are stimulating libido [9]. Regarding to different perspectives, their perception can be variable. During history, aphrodisiac agents have been used as stimulant of sexual intercourse, pleasure booster, flaming agents of libido and an agent to strip from shyness. Different countries from all around the world, have formed their own culture with various plants, according to the knowledge transferred from their ancestors. They adapted those plants into their traditional medicinal system [4]. There are different ways to use them, such as cigarette, tea, incense, pill, spice or meal, ointment and oil. In folkloric medicine of Chinese, Indian, Egyptian, Roman, Anatolian and Greek cultures, there are many different herbal and animal remedies [4-10]. The most common used plants or drugs are Cannabis sativa, opium, Panax ginseng, Ginkgo biloba, Zingiber officinale, Epimedium sp., Mucuna pruriens, Corynanthe yohimbe, Chelidonium majus, Tribulus terrestris, Crocus sativus, Phoenix dactylifera, Pegalum harmala, Catha edulis, Salvia haematodes, Lepidium meyenii, Asparagus racemosus, Glycyrrhiza glabra. Various foods also have been used as aphrodisiac agent such as vegetables [artichokes, asparagus), spices (anise, basil, coriander, fennel, sage, ginger, garlic, and saffron), oysters, chocolate, and fruits [10-14].

How to cite this article: Tufan S, Gülsoy Toplan G, Mat A. Ethnobotanical usage of plants as aphrodisiac agents in Anatolian folk medicine. Marmara Pharm J. 2018; 22 (2): 142-151. 
There are several ethnopharmacological reports on the medicinal uses of aphrodisiac plants of various cultures. Some of these plants or animal products were investigated for their aphrodisiac activity. The potential aphrodisiac properties were examined using in vitro assays, different animal models or clinical trials [11-14]. Although most studies demonstrated positive aphrodisiac activities, further studies are needed to reveal their mechanism of activity.

Ambrein and Panax ginseng have been shown to relax corpus cavernous smooth muscle or other smooth muscles in animal models, in vitro assays [15-16]. Hydro-alcoholic and aqueous extracts of Asparagus racemosus (400 mg/ kg body weight) have showed aphrosidiac properties on male wistar albino rats and these extracts have increased number of mounts and mating performance, and lower dose (200mg/ $\mathrm{kg}$ body weight) of hydro-alcoholic extract have shown moderate activity. In addition, $200 \mathrm{mg} / \mathrm{kg}$ body weight of milk decoction of A.racemosus roots has shown significant aphrodisiac effect. It increased mount frequency and caused decrease in mount latency, post ejaculatory latency and ejaculation latency [17]. There is one study showing that aqueous extract of Glycyrrhiza glabra roots and rhizomes have significant decreasing effects on mount latency and intromission latency when $150 \mathrm{mg} / \mathrm{kg} \& 300 \mathrm{mg} / \mathrm{kg}$ body weight/day administrated orally by gavage for 28 days on sexually active male rats [18]. Tribulus terrestris, also known as "ghokhru" and its extracts have been used as medicine traditionally in both Asia and India and also in Turkey to treat urinary, cardiovascular and gastrointestinal disorders. It is also, used by humankind to stimulate sexual desires due to its steroidal saponin protodioscin [11-19]. T. terrestris has been shown to increase erection quality in animals by observing an increased intracavernous pressure, while yohimbine, ginseng, and saffron increased erection quality in humans [11-20]. Saffron is one of the most expensive and highly valued spices in the world. The effect of Crocus sativus (saffron) on male erectile dysfunction was studied for 10 days, researchers followed up 20 male patients with erectile dysfunction whereby they were administered a tablet containing $200 \mathrm{mg}$ of saffron every morning. The selected patients were subjected to the nocturnal penile tumescence (NPT) test and the international index of erectile function questionnaire (IIEF-15) at the beginning and the end of the 10 days treatment. The results showed a statistically significant improvement after 10 days of taking saffron [21-22]. Passiflora incarnata has been used as anxiolytic, sedative, anticonvulsant all around the world. According to Dhawan et al. studies, the methanolic extract of Passiflora incarnata leaves showed strong aphrodisiac effects [23].

Besides, some of plants are usually used as aphrodisiac agents due to their strong antioxidant activities. Oxidative stress or excessive production of free radicals can damage sexual hormones and also decreased libido. This damage can cause infertility and decrease libido in both women and men. Recently, the studies revealed that lipid peroxidation plays significant role at infertility especially affecting sexual hormones, sperm motility and viability. In order to understand the relation between antioxidant and aphrodisiac activities, the effects of many plant extracts were examined by several researchers. According to these studies, there is a strong correlation between plants antioxidant properties and aphrodisiac activities [24].

Several plants have been used traditionally for centuries in Turkey for the treatment of various sexual disorders. Most of the remedies are prepared from a mixture of several species while there are some strong remedies with only one component.

The most popular traditional aphrodisiac is 'Mesir Macunu' (Mesir Paste). It is a traditional Turkish gum like candy produced in Manisa (Aegean region of Turkey). The original Mesir paste, prepared from 41 different spices, sugar and honey, is a tradition in Anatolia since $16^{\text {th }}$ century [25]. The majority of historians claims its similarities with theriac. The preparation of mesir macunu and its ceremonial distribution are still alive in Manisa [26, 27]. Today the content of mesir paste, given in Table 1, has minor differences from the original one. When the Mesir Paste is examined, it is clearly seen that has high antioxidant activity [18-28-2930-31-32]. The consist of Mesir paste includes many plants which possess strong antioxidant activity There are several studies on these plants antioxidant activities. Some these studies are given in Table 1. 
Table 1. Mesir paste.

\begin{tabular}{|c|c|c|c|c|c|}
\hline Local Name & Plant & Family & Part Used & References & Antioxidant \\
\hline Anason & Pimpinella anisum L. & Umbelliferae & Ripe Fruits & {$[11-33-34]$} & [28-35] \\
\hline Çivitotu & Isatis tinctoria $\mathrm{L}$. & Fabaceae & Leaves & [33] & [29-36] \\
\hline $\begin{array}{l}\text { Çöpçini / Çin } \\
\text { Saparması }\end{array}$ & Smilax china $\mathrm{L}$. & Liliaceae & Roots & [33] & [37-38] \\
\hline Çörekotu & Nigella sativa L. & Ranunculaceae & Sun Dried Seeds & [33] & [39-40] \\
\hline Darifülfül & Piper longum L. & Piperaceae & Bitter Fruits & [33] & {$[41-42]$} \\
\hline Defne & Laurus nobilis L. & Lauraceae & Leaves & [33] & [43-44] \\
\hline $\begin{array}{c}\text { Galanya / Kalanga / } \\
\text { Büyük Havlican }\end{array}$ & Alpinia galangal $\mathrm{L}$. & Zingiberaceae & Rhizomes & [33] & {$[45-46]$} \\
\hline $\begin{array}{l}\text { Hindistan } \\
\text { Cevizi/Kopra }\end{array}$ & Cocus nucifera L. & Arecaceae & Dried Seeds & [33-47] & {$[48]$} \\
\hline Hindistan Çiçeği & Myristica fragrans Houtt. & Myristicaea & Flowers & [12-14-33-49] & {$[50-51]$} \\
\hline Hıyarşenbe & Cassia fistula L. & Fabaceae & Ripe Fruits & [33] & {$[52-53]$} \\
\hline Kakule & Elettaria cardamomum L. & Zingiberaceae & $\begin{array}{l}\text { Dried Unripe } \\
\text { Fruits }\end{array}$ & [33] & [54-55] \\
\hline Kara Halile & Terminalia chebula Retz. & Combretaceae & Fruits & [33] & [56-67] \\
\hline Karabiber & Piper nigrum L. & Piperaceae & $\begin{array}{l}\text { Dried Unripe } \\
\text { Fruits }\end{array}$ & [33] & [58-59] \\
\hline Karanfil & Syzygium aromaticum L. & Myrtaceae & Flowers & [11-12-33-49] & [60-61] \\
\hline $\begin{array}{l}\text { Kebabiye / Kebabe } \\
\text { / Kübabe }\end{array}$ & Piper cubeba L. & Piperaceae & $\begin{array}{l}\text { Dried Unripe } \\
\text { Fruits }\end{array}$ & [33] & [30] \\
\hline Kimyon & Cuminum cyminum L. & Umbelliferae & Dried Ripe Fruits & [33] & [62-63] \\
\hline Kişniş & Coriandrum satioum $\mathrm{L}$. & Umbelliferae & Fruits & [11-33-34] & [64-65] \\
\hline Küçük Havlican & $\begin{array}{c}\text { Galanga officinalis (L.) } \\
\text { Wild. }\end{array}$ & Zingiberaceae & Dried Rhizomes & [33] & {$[-]$} \\
\hline Mercanköşk & Origanum majorana L. & Labiatae & Aerial parts & [33] & [66-67] \\
\hline Meyan & Glycyrrhiza glabra L. & Leguminosae & $\begin{array}{l}\text { Condensed Root } \\
\text { Extract }\end{array}$ & [33] & [31-68] \\
\hline Mirsafi & $\begin{array}{l}\text { Commiphora myrrha } \\
\text { (Nees) Engl. }\end{array}$ & Burceraceae & Usare & [33] & [32-69] \\
\hline Portakal & $\begin{array}{c}\text { Citrus aurantium var. } \\
\text { dulce } \mathrm{L} \text {. }\end{array}$ & Rutaceae & Pericarpium & [33] & {$[-]$} \\
\hline Ravent & Rheum officinale L. & Polygonaceae & Rhizomes & [33] & [70] \\
\hline Rezene & Foeniculum vulgare Mill. & Umbelliferae & Dried Ripe Fruits & [11-33] & [71-72] \\
\hline Safran & Crocus sativus L. & Iridaceae & Stigma & [11-12-14-33] & {$[73-74]$} \\
\hline Sakız & Pistacia lentiscus L. & Anacardiaceae & Resin & [33] & {$[75-76]$} \\
\hline Sarı Halile & $\begin{array}{l}\text { Terminalia citrina } \\
\text { (Gaertn.) Roxb. ex } \\
\text { Fleming }\end{array}$ & Combretaceae & Fruits & [33] & [77] \\
\hline Sinameki & Cassia acutifolia Mill. & Leguminosae & Dried Leaves & [33] & [78] \\
\hline Siyah Hardal & Brassica nigra L. & Cruciferae & Seeds & [33] & [79] \\
\hline Tarçın & $\begin{array}{l}\text { Cinnamomum cassia (L.) } \\
\text { J.Presl. }\end{array}$ & Lauraceae & Cortex & [33] & [80-81] \\
\hline Tarçın Çiçeği & $\begin{array}{c}\text { Cinnamomum zeylanicum } \\
\text { L. }\end{array}$ & Lauraceae & Flowers & [33] & [82] \\
\hline Teke mersini & Vaccinium myrtillus L. & Ericaceae & Fruits & [33] & [83] \\
\hline Topalak & Cyclamen coum Miller & Primulaceae & Tubers & [33] & {$[-]$} \\
\hline $\begin{array}{l}\text { Udülkahr / } \\
\text { Nezleotu }\end{array}$ & Anacylus pyrethrum L. & Asteraceae & Dried Roots & [33] & {$[-]$} \\
\hline Vanilya & Vanilla planifolia Andr. & Orchidaceae & Fermented Fruit & [33] & [84-85] \\
\hline Yenibahar & Pimenta officinalis L. & Myrtaceae & $\begin{array}{l}\text { Dried Unripe } \\
\text { Fruits }\end{array}$ & [33] & {$[-]$} \\
\hline Zencefil & Zingiber officinale Rosc. & Zingiberaceae & Rhizomes & [11-33] & [86-87] \\
\hline $\begin{array}{l}\text { Zerdeçal / } \\
\text { Hintsafranı / } \\
\text { Sarıboya }\end{array}$ & Curcuma longa $\mathrm{L}$. & Zingiberaceae & Rhizomes & [33] & [88-89] \\
\hline Zulumba & Curcuma zedoaria Rosc. & Zingiberaceae & Rhizomes & [33] & [90] \\
\hline
\end{tabular}




\section{MATERIALS AND METHODS}

Ethnobotanical studies and thesis about Anatolian folk medicine were rewieved and plants used as aphrodisiac were selected. Information about them were reported. Among all studies, the plants used as aphrodisiac agent in more than one publication have been selected and added to the Table 2 .

Table 2. The list of Turkish aphrodisiac plants.

\begin{tabular}{|c|c|c|c|c|c|c|}
\hline Plant & Local Name & Family & Part Used & Preparation & Dosage & References \\
\hline $\begin{array}{c}\text { Prangos ferulacea } \\
\text { L. }\end{array}$ & $\begin{array}{l}\text { Çağşır, } \\
\text { köfteotu, } \\
\text { kürdanotu, } \\
\text { melekotu, } \\
\text { pitrak }\end{array}$ & Apiaceae & Roots & $\begin{array}{l}\text { Mixed with } \\
\text { sweets }\end{array}$ & & [91] \\
\hline $\begin{array}{c}\text { Prangos } \\
\text { meliocarpoides } \\
\text { Boiss. var. } \\
\text { meliocarpoides }\end{array}$ & $\begin{array}{l}\text { Çarșır, hiltil, } \\
\text { sultan teresi }\end{array}$ & Apiaceae & Roots & $\begin{array}{l}\text { Mixed with } \\
\text { sweets }\end{array}$ & & [91] \\
\hline Morina persica $\mathrm{L}$. & Boğa dikeni & Morinaceae & $\begin{array}{l}\text { Aerial parts, } \\
\text { Roots }\end{array}$ & Eaten raw & & [91] \\
\hline $\begin{array}{c}\text { Hippophae } \\
\text { rhamnoides L. } \\
\text { subsp. caucasica }\end{array}$ & Pişot & Elaeagnaceae & Fruits & Eaten raw & & [92] \\
\hline $\begin{array}{l}\text { Brassica oleracea } \mathrm{L} \text {. } \\
\text { var. capitata L. }\end{array}$ & Başlı lahana & Cruciferae & Seeds & & & [93] \\
\hline $\begin{array}{l}\text { Corylus avellana } \mathrm{L} . \\
\text { var. avellana } \\
\text { Corylus colurna L. } \\
\text { Corylus maxima } \\
\text { Miller }\end{array}$ & Findık & Corylaceae & Seeds & $\begin{array}{l}1 \mathrm{~kg} \text { of hazelnut, } \\
\text { butter, honey, } \\
\text { raisins and } 0.5 \\
\mathrm{~kg} \text { of garlic are } \\
\text { pounded and } \\
\text { mixed. }\end{array}$ & $\begin{array}{l}\text { Mixture eaten } \\
\text { every } \\
\text { morning on } \\
\text { empty } \\
\text { stomach. }\end{array}$ & [93-94] \\
\hline $\begin{array}{c}\text { Junierus drupacea } \\
\text { L. }\end{array}$ & And1z & Cupressaceae & Cortex & $\begin{array}{l}\text { Decoction, } \\
\text { Powder }\end{array}$ & & [95] \\
\hline Orchis spp. L. & Salep & Orchidaceae & Tubers & Powder & & [95] \\
\hline Cerasus mahalep L. & Mahlep & Rosaceae & Seeds & $\begin{array}{l}\text { Powder, } \\
\text { Infusion }\end{array}$ & & [95-96] \\
\hline $\begin{array}{c}\text { Capparis spinosa L. } \\
\text { var. spinosa }\end{array}$ & Gebere & Capparaceae & Seeds & Crushed Extract & & [97] \\
\hline $\begin{array}{c}\text { Matricaria } \\
\text { chamomilla L. }\end{array}$ & $\begin{array}{l}\text { Papatya, deli } \\
\text { papatya, } \\
\text { tellipapatya }\end{array}$ & Asteraceae & Capitulum & Infusion & & [97] \\
\hline Rosa canina L. & $\begin{array}{l}\text { Kus,burnu, } \\
\text { itburnu }\end{array}$ & Rosaceae & $\begin{array}{l}\text { Fruits, } \\
\text { Roots, } \\
\text { Seeds }\end{array}$ & $\begin{array}{l}\text { Decoction, } \\
\text { Fruits (eaten by } \\
\text { chewing), Raw, } \\
\text { Mixture (jam } \\
\text { etc.) }\end{array}$ & $\begin{array}{c}\text { Herbal tea } \\
\text { with hibiscus } \\
\text { 1-2 glass per } \\
\text { day for 2-3 } \\
\text { weeks. } \\
\text { Paste } \\
\text { prepared } \\
\text { from fresh } \\
\text { fruits }(2-3 \mathrm{~kg}) \\
\text { and paste } \\
\text { mixed with } \\
\text { honey in the } \\
\text { raito of 1:3. } \\
\text { Consumed on } \\
\text { empty } \\
\text { stomach. }\end{array}$ & $\begin{array}{c}\text { [98-99-100-101- } \\
102-103]\end{array}$ \\
\hline Ceratonia siliqua L. & $\begin{array}{l}\text { Keçiboynuzu, } \\
\text { Harnup }\end{array}$ & Fabaceae & Fruits & $\begin{array}{l}\text { Mixture (jam } \\
\text { etc.), Fruits } \\
\text { (eaten by } \\
\text { chewing), Raw, } \\
\text { Decoction, Fruit } \\
\text { powder, Spice }\end{array}$ & $\begin{array}{c}\text { 2-3 pieces or } \\
2-3 \\
\text { tablespoons } \\
\text { are eaten } \\
\text { before } \\
\text { breakfast for } \\
\text { 2-3 weeks. }\end{array}$ & [98] \\
\hline
\end{tabular}


Table 3 (Continued). The list of Turkish aphrodisiac plants.

\begin{tabular}{|c|c|c|c|c|c|c|}
\hline Plant & Local Name & Family & Part Used & Preparation & Dosage & References \\
\hline $\begin{array}{l}\text { Ferula elaeochytris } \\
\text { Korovin }\end{array}$ & $\begin{array}{l}\text { Çarşır, çaaşır, } \\
\text { çarşır otu, } \\
\text { çakşır, çarşır } \\
\text { göbeği }\end{array}$ & Apiaceae & $\begin{array}{l}\text { Roots, } \\
\text { Flowers, } \\
\text { Seeds, } \\
\text { Fruits }\end{array}$ & $\begin{array}{l}\text { Spice, Rhizome } \\
\text { juice, Fruits } \\
\text { (eaten by } \\
\text { chewing), } \\
\text { Powdered roots }\end{array}$ & $\begin{array}{c}\text { Drunken } 1 \\
\text { teacup twice a } \\
\text { day for 2-3 } \\
\text { weeks. } \\
\text { Peeled roots } \\
\text { were dried, } \\
\text { pulverized } \\
\text { and mixed } \\
\text { with honey. }\end{array}$ & [96-98-104] \\
\hline $\begin{array}{c}\text { Gundelia } \\
\text { tournefortii L. var. } \\
\text { tournefortii L. }\end{array}$ & $\begin{array}{l}\text { Kenger, } \\
\text { Hüznbol }\end{array}$ & Asteraceae & Latex, Roots & $\begin{array}{l}\text { A piece of latex } \\
\text { is eaten, } \\
\text { Decoction }\end{array}$ & & [100-103-105] \\
\hline Zea mays L. & Misir & Poaceae & Style & $\begin{array}{l}\text { Decoction, } \\
\text { Infusion }\end{array}$ & & [100-105-106] \\
\hline $\begin{array}{c}\text { Ferula communis } \\
\text { L. }\end{array}$ & $\begin{array}{l}\text { Çarşır, çarşır } \\
\text { otu, çakşır, } \\
\text { çarşır göbeği }\end{array}$ & Apiaceae & Roots & Mash & $\begin{array}{l}1 \text { teaspoon } \\
\text { twice a day } \\
\text { for } 1-2 \text { weeks }\end{array}$ & [107] \\
\hline $\begin{array}{l}\text { Opuntia ficus- } \\
\text { indica L. }\end{array}$ & $\begin{array}{l}\text { Kaynana dili, } \\
\text { Frenk inciri }\end{array}$ & Cactaceae & Fruits & $\begin{array}{c}\text { Peeled and } \\
\text { eaten raw }\end{array}$ & & [105] \\
\hline $\begin{array}{c}\text { Elaeagnus } \\
\text { angustifolia L. }\end{array}$ & İğde & Elaeagnaceae & Flowers & Scent & & [105] \\
\hline $\begin{array}{l}\text { Calicotome villosa } \\
\text { (Poir.) Link }\end{array}$ & Azgan & Fabaceae & $\begin{array}{c}\text { Flowers, } \\
\text { Essential oil }\end{array}$ & Scent & & [105] \\
\hline $\begin{array}{c}\text { Sideritis syriaca L. } \\
\text { subsp. } \\
\text { Nusairiensis }\end{array}$ & Adaçayı & Lamiaceae & Aerial parts & Decoction & & [105] \\
\hline $\begin{array}{l}\text { Sesamum indicum } \\
\text { L. }\end{array}$ & Susam & Pedaliaceae & Seeds & Eaten raw & & [105] \\
\hline Pinus pinea L. & Çam fistığg & Pinaceae & $\begin{array}{l}\text { Seeds, } \\
\text { Fruits }\end{array}$ & Eaten raw & & [103-108] \\
\hline $\begin{array}{c}\text { Arceuthos drupacea } \\
\text { Ant. et Kotschy. }\end{array}$ & Andiz & Cupressacaceae & $\begin{array}{c}\text { Grape } \\
\text { molasses }\end{array}$ & & & [108] \\
\hline Urtica dioica $\mathrm{L}$. & Isırgan & Urticaceae & $\begin{array}{l}\text { Aerial parts, } \\
\text { Seeds, } \\
\text { Leaves }\end{array}$ & $\begin{array}{l}\text { Infusion, } \\
\text { Crushed, Leaves } \\
\text { mixed with } \\
\text { sweets }\end{array}$ & & [91] \\
\hline $\begin{array}{c}\text { Melilotus } \\
\text { officinalis L. }\end{array}$ & Kokulu yonca & Fabaceae & Aerial parts & Decoction & & [92] \\
\hline $\begin{array}{c}\text { Punica granatum } \\
\text { L. }\end{array}$ & Nar & Punicaceae & Seeds & Eaten raw & & [109] \\
\hline
\end{tabular}

\section{RESULTS AND DISCUSSION}

According to ethnobotanical studies, 27 medicinal plants are listed as aphrodisiacs in Turkish traditional medicine. These plants are given in Table 2. The most popular plant is Rosa canina which is widely used through all Anatolia. The majority of the plants are prepared in the form of infusion or decoction from freshly collected plant parts.

Mesir paste is remarkably the famous traditional aphrodisiac since centuries.

While comparing usage methods of aphrodisiacs in Turkey and other countries, similarities and differences were observed. For example, Rosa canina fruits mixed with honey are used in Turkey while in other countries its essential oil is reputed as aphrodisiac. The flowers of Elaeagnus angustifolia are recognized as aphrodisiac in Turkish ethnobotanical studies in Iran its fruits are used as female aphrodisiac. Sesamum indicum seeds are used against impotency in both Turkey and India. Turkish people prefer eating raw seeds for treatment. Ayurvedic system suggests a mixture of seeds with honey once a day, before meal. Urtica dioica, a component of Swiss love potion, is also used in sweet mixtures in Turkey for the same purpose. Brassica oleracea, Corylus avellana, Capparis spinosa have similar aphrodisiac usage in different countries. Although Lepidium meyenii is quite popular as aphrodisiac plants in other countries, Lepidium sativum have been used as aphrodisiac agent only in Capadocia region from Turkey [110]. 
The adverse effects and expensive price of synthetic drugs orient the humankind to natural treatment. Natural products have been used for centuries and investigations proved the effectiveness of some of them.

Author contributions: Concept - S.T.,G.G.T.; Design - S.T.,G.G.T., A.M.; Supervision - A.M.; Resource - S.T., G.G.T.; Materials - S.T., G.G.T.; Data Collection and/or Processing S.T.,G.G.T.; Analysis and/or Interpretation S.T.,G.G.T.,A.M.; Literature Search - S.T.,G.G.T.; Writing - S.T.,G.G.T. A.M.; Critical Reviews - S.T.,G.G.T., A.M.

Conflict of interest statement: The authors declared no conflict of interest.

\section{REFERENCES}

[1] Chauhan NS, Sharma V, Dixit VK, Thakur M. A review on plants used for improvement of sexual performance and virility. Biomed Res Int. 2014; 2014: 1-19.

[2] Vernant JP. Evren, Tanrılar, İnsanlar. Dost Kitabevi Yayınları, Ankara. 2001.

[3] Daly KN. Greek and Roman Mythology A to Z. Chelsea House Publishers, New York. 2009.

[4] Rätsch C. Plants of Love: Aphrodisiacs in Myth, History, and the Present. Ten Speed Press, Berkeley. 1997.

[5] Babacan SS. İnsan Seksüalitesinin Kültürel ve Psikososyal Yönleri. Kefdergi. 2003; 11: 131-136.

[6] İncesu C. Cinsel İşlevler ve Cinsel İşlev Bozuklukları. J Clin Psy. 2004; 7(3): 3-13.

[7] Vural AG. Evlilik öncesi cinsel danışmanlık programının yeni evli çiftlerin cinsel doyumuna etkisi. T.C. Ege Üniversitesi Saglık Bilimleri Enstitüsü, İzmir. 2007.

[8] Sadock BJ. Normal İnsan Cinselliği, Cinsel İşlev ve Cinsel Kimlik Bozuklukları. Güneş, Ankara. 2007.

[9] Mathur, M. Herbal aphrodisiac their need, biology and status: Global and regional scenario. J Nat Prod. 2012; 5: 131-146.

[10] Rätsch C, Müller-Ebeling C. The Encyclopedia of Aphrodisiacs Psychoactive Substances for Use in Sexual Practices. Park Street Press, Toronto. 2003.

[11] Melnyk JP, Marcone MF. Aphrodisiacs from plant and animal sources - A review of current scientific literature. Food Res Int. 2011; 4: 840-850.

[12] Sumalatha K, Kumar SA, Lakshmi SM. Review on natural aphrodisiac potentials to treat sexual dysfunction. IJPT. 2010; 1(1): 6-14.

[13] Thakur M, Chauhan NS, Bhargava A, Dixit VK. A comparative study on aphrodisiac activity of some Ayurvedic herbs in male albino rats. Arch Sex Behav. 2009; 38 (6): 1009-1015.

[14] Singh R, Ali A, Gupta G, Semwal A, Jeyabalan G. Some medicinal plants with aphrodisiac potential: A current status. J Acute Dis. 2013; 2(3): 179-188.

[15] Taha SA, Raza M, El-Khawad IE. Effect of ambrein on smooth muscle responses to various agonists. J Ethnopharmacol. 1998; 60(1): 19-26.

[16] Kim HJ, Woo DS, Lee G, Kim, JJ. The relaxation effects of ginseng saponin in rabbit corporal smooth muscle: Is it a nitric oxide donor? Br J Urol. 1998; 82(5): 744-748.

[17] Wani JA, Achur RN, Nema RK. Phytochemical screening and aphrodisiac property of Asparagus racemosus. Int J Pharm Drug Res. 2011; 3(2): 112-115.

[18] Awate SA, Patil RB, Ghode PD, Patole V, Pachauri D, Sherief SH. Aphrodisiac activity of aqueous extract of Glycyrrhiza glabra in male wistar rats. World J Pharm Res. 2012; 1(2): 371-378.

[19] Arcasoy HB, Erenmemisoglu A, Tekol Y, Kurucu S, Kartal M. Effect of Tribulus terrestris L. saponin mixture on some smooth muscle preparations: a preliminary study. Boll Chim Farm. 1998; 137(11) 473-475.

[20] Singh S, Gupta YK. Aphrodisiac activity of Tribulus terrestris Linn. in experimental models in rats. J Men Health's. 2011; 8(1): S75- S77.

[21] Hosseinzadeh H, Ziaee T, Sadeghi A. The effect of saffron, Crocus sativus stigma, extract and its constituents, safranal and crocin on sexual behaviors in normal male rats. Phytomedicine. 2008; 15(6): 491-495.

[22] Shamsa A, Hosseinzadeh H, Molaei M, Shakeri M T, Rajabi O. Evaluation of Crocus sativus L.(saffron) on male erectile dysfunction: a pilot study. Phytomedicine. 2008; 16(8):690-693. 
[23] Dhawan K, Kumar S,Sharma A. Aphrodisiac activity of methanol extract of leaves of Passiflora incarnata Linn. in mice. Phytother Res. 2003: 17(4); 401-403.

[24] Muanya CA, Odukoya OA. Lipid peroxidation as index of activity in aphrodisiac herbs. J Plant Sci. 2008; 3(1): 9298.

[25] Baylav N. Eczacılık Tarihi. Yörük Matbaası, İstanbul. 1968.

[26] Nicolas M. Un électuaire de jouvence et une fête de printemps à Manisa (Turquie). In: Le Cuisinier et le Philosophe, Hommage à Maxime Rodinson, Maisonneuve et Larose. Paris. 1982, pp. 71-80.

[27] Şimsek F. Mithridaticum and Mesir: The Story of an Antidote from Antiquity into Ottoman Times. MJH. 2012; 2(2): 243-250.

[28] Hinneburg I, Dorman HJD, Hiltunen R. Antioxidant activities of extracts from selected culinary herbs and spices. Food Chem. 2006; 97(1): 122-129.

[29] Li HB, Wong CC, Cheng KW, Chen F. Antioxidant properties in vitro and total phenolic contents in methanol extracts from medicinal plants. LWT1. 2008; 41(3): 385-390.

[30] Aqil F, Ahmad I, Mehmood Z. Antioxidant and free radical scavenging properties of twelve traditionally used Indian medicinal plants. Turk J Biol. 2006; 30: 177-183.

[31] Wojdyło A, Oszmiański J, Czemerys R. Antioxidant activity and phenolic compounds in 32 selected herbs. Food Chem. 2007; 105(3): 940-949.

[32] Assimopoulou A, Zlatanos S, Papageorgiou V. Antioxidant activity of natural resins and bioactive triterpenes in oil substrates. Food Chem. 2005; 92(4): 721-727.

[33] Güven AN. Master Thesis. Mesir macununun antioksidan aktivitesinin ve reolojik özelliklerinin belirlenmesi. Department of Food Engineering, Faculty of Science, İstanbul Technical University, Maslak, İstanbul, Turkey, 2010.

[34] Martins N, Barros L, Santos-Buelga C, Ferreira ICFR. Antioxidant potential of two Apiaceae plant extracts: A comparative study focused on the phenolic composition. Ind Crops Prod. 2016; 79: 188-194.

[35] Gülçın İ, Oktay M, Kıreçcı E, Küfrevığlu Öİ. Screening of antioxidant and antimicrobial activities of anise (Pimpinella anisum L.) seed extracts. Food Chem. 2003; 83(3): 371-382.

[36] Peschel W, Sánchez-Rabaneda F, Diekmann W, Plescher A, Gartzía I, Jiménez D, Lamuela-Raventósc R, Buxaderas $\mathrm{S}$, Codina C. An industrial approach in the search of natural antioxidants from vegetable and fruit wastes. Food Chem. 2006; 97(1): 137-150.

[37] Lee SE, Ju EM, Kim JH. Free radical scavenging and antioxidant enzyme fortifying activities of extracts from Smilax china root. Exp Mol Med. 2001; 33(4): 263-268.

[38] Kim KK, Kang YH, Kim DJ, KIM TW, Choe M. Comparison of antioxidant, a-glucosidase inhibition and antiinflammatory activities of the leaf and root extracts os Similax china L. J Nutr Health. 2013; 46 (4): 315-323.

[39] Burits M, Bucar F. Antioxidant activity of Nigella sativa essential oil. Phytother Res. 2000; 14(5): 323-328.

[40] Erkan N, Ayranci G, Ayranci E. Antioxidant activities of rosemary (Rosmarinus officinalis L.) extract, blackseed (Nigella sativa L.) essential oil, carnosic acid, rosmarinic acid and sesamol. Food Chem. 2008; 110(1): 76-82.

[41] Khalaf NA, Shakya AK, Al-Othman A , El-Agbar Z , Farah H. Antioxidant activity of some common plants. Turk J Biol. 2008; 32: 51-55.

[42] Reddy N, Nagoor Vali D, Rani M, Rani S. Evaluation of antioxidant, antibacterial and cytotoxic effects of green synthesized silver nanoparticles by Piper longum fruit. Mater Sci Eng C Mater Biol App. 2014; 34: 115-122.

[43] Škerget M, Kotnik P, Hadolin M, Hraš AR, Simonič M, Kneza Ž. Phenols, proanthocyanidins, flavones and flavonols in some plant materials and their antioxidant activities. Food Chem. 2005; 89(2): 191-198.

[44] Ferreira A, Proença C, Serralheiro MLM, Araújo MEM. The in vitro screening for acetylcholinesterase inhibition and antioxidant activity of medicinal plants from Portugal. J Ethnopharmacol. 2006; 108(1): 31-37.

[45] Juntachote T, Berghofer E. Antioxidative properties and stability of ethanolic extracts of Holy basil and Galangal. Food Chem. 2005; 92(2): 193-202.

[46] Mayachiew P, Devahastin S. Antimicrobial and antioxidant activities of Indian gooseberry and galangal extracts. LWT. 2008; 41(7): 1153-1159. 
[47] Shen X, Chen W, Zheng Y, Lei X, Tang M, Wang H, Song F. Chemical composition, antibacterial and antioxidant activities of hydrosols from different parts of Areca catechu L. and Cocos nucifera L. Ind Crops Prod. 2017; 96: 110119.

[48] Chakraborty M, Mitra A. The antioxidant and antimicrobial properties of the methanolic extract from Cocos nucifera mesocarp. Food Chem. 2008; 107(3): 994-999.

[49] Tajuddin, Ahmad S, Latif A, Qasmi IA. Aphrodisiac activity of 50\% ethanolic extracts of Myristica fragrans Houtt. (nutmeg) and Syzygium aromaticum (L) Merr. \& Perry (clove) in male mice: a comparative study. ISCMR. 2003; 3(6): $1-5$.

[50] Chatterjee S, Niaz Z, Gautam S, Adhikari S, Variyar P, Sharma A. Antioxidant activity of some phenolic constituents from green pepper (Piper nigrum L.) and fresh nutmeg mace (Myristica fragrans). Food Chem. 2007; 101(2): 515-523.

[51] Singh G, Marimuthu P, de Heluani CS, Catalan C. Antimicrobial and antioxidant potentials of essential oil and acetone extract of Myristica fragrans Houtt. (Aril Part). J Food Sci. 2005; 70(2): M141-M148.

[52] Siddhuraju P, Mohan P, Becker K. Studies on the antioxidant activity of Indian Laburnum (Cassia fistula L.): a preliminary assessment of crude extracts from stem bark, leaves, flowers and fruit pulp. Food Chem. 2002; 79(1): 61-67.

[53] Luximon-Ramma A, Bahorun T, Soobrattee MA, Aruoma OI. Antioxidant activities of phenolic, proanthocyanidin, and flavonoid components in extracts of Cassia fistula. J Agric Food Chem. 2002; 50(18): 5042-5047.

[54] Verma SK, Jain V, Katewa SS. Blood pressure lowering, fibrinolysis enhancing and antioxidant activities of Cardamom (Elettaria cardamomum). Indian J Biochem Biophys. 2009; 46: 503-506.

[55] Singh G, Kiran S, Marimuthu P, Isidorov V, Vinogorova V. Antioxidant and antimicrobial activities of essential oil and various oleoresins of Elettaria cardamomum (seeds and pods). J Sci Food Agr. 2008; 88(2): 280-289.

[56] Hazra B, Sarkar R, Biswas S, Mandal N. Comparative study of the antioxidant and reactive oxygen species scavenging properties in the extracts of the fruits of Terminalia chebula, Terminalia belerica and Emblica officinalis. BMC Complement Altern Med. 2010; 10(20); 1-15.

[57] Cheng HY, Lin TC, Yu KH, Yang CM, Lin CC. Antioxidant and Free Radical Scavenging Activities of Terminalia chebula. Biol Pharm Bul. 2003; 26: 1331-1335.

[58] Vijayakumar RS, Surya D, Nalini N. Antioxidant efficacy of black pepper (Piper nigrum L.) and piperine in rats with high fat diet induced oxidative stress. Redox Rep. 2004; 9(2): 105-110.

[59] Gülçin İ. The antioxidant and radical scavenging activities of black pepper (Piper nigrum) seeds. Int J Food Sci Nutr. 2005; 56(79): 491-499.

[60] Lee K, Shibamoto T. Antioxidant property of aroma extract isolated from clove buds [Syzygium aromaticum (L.) Merr. et Perry]. Food Chem. 2001; 74(4): 443-448.

[61] Atawodi SE, Atawodi, JC, Pfundstein B, Spiegelhalder, Bartsch Hand, Owen R. Assessment of the polyphenol components and in vitro antioxidant properties of Syzygium aromaticum (L.) Merr.\& Perry. EJEAFChe. 2011; 10(3): 1970-1978.

[62] El-Ghorab AH, Nauman M, Anjum FM, Shahzad Hussain S, Nadeem M. A Comparative study on chemical composition and antioxidant activity of ginger (Zingiber officinale) and cumin (Cuminum cyminum). J Agric Food Chem. 2010; 58(14): 8231-8237.

[63] Rebey IB, Jabri-Karoui I, Hamrouni-Sellami I, Bourgou S, Limam F, Marzouk B. Effect of drought on the biochemical composition and antioxidant activities of cumin (Cuminum cyminum L.) seeds. Ind Crops Prod. 2012; 36(1): $238-245$.

[64] Ramadan MF, Kroh LW, Mörsel JT. Radical scavenging activity of black cumin (Nigella sativa L.), coriander (Coriandrum sativum L.), and niger (Guizotia abyssinica Cass.) crude seed oils and oil fractions. J Agric Food Chem. 2003; 51(24): 6961-6969.

[65] Samojlik I, Lakić N, Mimica-Dukić N, Đaković-Švajcer K, Božin B. Antioxidant and hepatoprotective potential of essential oils of coriander (Coriandrum sativum L.) and caraway (Carum carvi L.) (Apiaceae). J Agric Food Chem. 2010; 58(15): 8848-8853.

[66] Roby M, Sarhan M, Selim K, Khalel K. Evaluation of antioxidant activity, total phenols and phenolic compounds in thyme (Thymus vulgaris L.), sage (Salvia officinalis L.), and marjoram (Origanum majorana L.) extracts. Ind Crops Prod. 2013; 43: 827-831. 
[67] Economou KD, Oreopoulou V, Thomopoulos CD. Antioxidant activity of some plant extracts of the family Labiatae. J Am Oil Chem Soc. 1991; 68 (2): 109-113.

[68] Visavadiya NP, Soni B, Dalwadi N. Evaluation of antioxidant and anti-atherogenic properties of Glycyrrhiza glabra root using in vitro models. Int J Food Sci Nutr. 2009; 60(2): 135-149.

[69] Mohamed A, Ali S, EL-Baz F, Hegazy A, Kord M. Chemical composition of essential oil and in vitro antioxidant and antimicrobial activities of crude extracts of Commiphora myrrha resin. Ind Crops Prod. 2014; 57: 10-16.

[70] Yizhong, Sun M, Xing J, Corke H. Antioxidant phenolic constituents in roots of Rheum officinale and Rubia cordifolia: Structure-radical scavenging activity relationships. J Agric Food Chem. 2004; 52(26): 7884-7890.

[71] Oktay M, Gülçin İ, Küfrevioğlu Öİ. Determination of in vitro antioxidant activity of fennel (Foeniculum vulgare) seed extracts. Food Sci Technol. 2003; 36(2): 263-271.

[72] Singh G, Maurya S, de Lampasona M, Catalan C. Chemical constituents, antifungal and antioxidative potential of Foeniculum vulgare volatile oil and its acetone extract. Food Control. 2006; 17(9): 745-752.

[73] Karimi E, Oskoueian E, Hendra R, Jaafar HZE. Evaluation of Crocus sativus L. Stigma phenolic and flavonoid compounds and its antioxidant activity. Molecules. 2010; 15(9): 6244-6256.

[74] Assimopoulou AN, Sinakos Z, Papageorgiou VP. Radical scavenging activity of Crocus sativus L. extract and its bioactive constituents. Phytother Res. 2005; 19(11): 997-1000.

[75] Gardeli C, Vassiliki P, Athanasios M, Kibouris T, Komaitis M. Essential oil composition of Pistacia lentiscus L. and Myrtus communis L.: Evaluation of antioxidant capacity of methanolic extracts. Food Chem. 2008; 107(3): 1120-1130.

[76] Barra A, Coroneo V, Dessi S , Cabras P , Angioni A. Characterization of the volatile constituents in the essential oil of Pistacia lentiscus L. from different origins and its antifungal and antioxidant activity. J Agric Food Chem. 2007; 55(17): 7093-7098.

[77] Palasuwan A, Soogarun S, Lertlum T, Pradniwat P, Wiwanitkit V. Inhibition of Heinz body induction in an in vitro model and total antioxidant activity of medicinal Thai plants. Asian Pacific J Cancer Prev. 2005; 6: 458-463.

[78] Laghari AQ, Memon S, Nelofar A, Laghari AH. Extraction, identification and antioxidative properties of the flavonoid-rich fractions from leaves and flowers of Cassia angustifolia. Am J Analyt Chem. 2011; 2: 871-878.

[79] Kim JW, Minamikawa T. Hydroxyl radical-scavenging effects of spices and scavengers from brown mustard (Brassica nigra). Biosci Biotechnol Biochem. 1997; 61(1): 118-123.

[80] Lin CC, Wu SJ, Chang CH, Ng LT. Antioxidant activity of Cinnamomum cassia. Phytother Res. 2003; 17(7): 726-730.

[81] Yang CH, Li RX, Chuang Ly. Antioxidant activity of various parts of Cinnamomum cassia extracted with different extraction methods. Molecules. 2012; 17(6): 7294-7304.

[82] Jayaprakasha GK, Rao LJM, Sakariah KK. Volatile constituents from Cinnamomum zeylanicum fruit stalks and their antioxidant activities. J Agric Food Chem. 2003; 51 (15): 4344-4348.

[83] Faria A, Oliveira J, Neves P, Gameiro P, Santos-Buelga C, de Freitas V, Mateus N. Antioxidant properties of prepared blueberry (Vaccinium myrtillus) extracts. J Agric Food Chem. 2005; 53(17): 6896-6902.

[84] Shyamala BN, Naidu MM, Sulochanamma G, Srinivas P. Studies on the antioxidant activities of natural vanilla extract and its constituent compounds through in vitro models. J Agric Food Chem. 2007; 55(19): 7738-7743.

[85] Kamat JP, Ghosh A, Devasagayam T. Vanillin as an antioxidant in rat liver mitochondria: Inhibition of protein oxidation and lipid peroxidation induced by photosensitization. Mol Cell Biochem. 2000; 209 (1): 47-53.

[86] Stoilova I, Krastanov A, Stoyanova A, Denev P, Gargova S. Antioxidant activity of a ginger extract (Zingiber officinale). Food Chem. 2007; 102(3): 764-770.

[87] Singh G, Kapoor I, Singh P, Heluani CS, Lampasona MP, Catalan CAN. Chemistry, antioxidant and antimicrobial investigations on essential oil and oleoresins of Zingiber officinale. Food Chem Toxicol. 2008; 46 (10): 3295-3302.

[88] Toda S, Miyase T, Arichi H, Tanizawa H, Takino Y. Natural antioxidants.III. Antioxidative components isolated from rhizome of Curcuma longa L. Chem Pharm Bull. 1985; 33; 1725-1728.

[89] Ramsewak R, DeWitt D, Nair M. Cytotoxicity, antioxidant and anti-inflammatory activities of Curcumins I-III from Curcuma longa. Phytomedicine. 2000; /(4): 303-308.

[90] Mau JL, Lai EYC, Wang NP, Chen CC, Chang CH, Chyau CC. Composition and antioxidant activity of the essential oil from Curcuma zedoaria. Food Chem. 2003; 82(4): 583-591. 
[91] Özdemir E, Alpınar K. An ethnobotanical survey of medicinal plants in western part of central Taurus Mountains: Aladaglar (Nigde - Turkey). J Ethnopharmacol. 2015; 166: 53-65.

[92] Cakilcioglu U, Khatun S, Turkoglu I, Hayta S. Ethnopharmacological survey of medicinal plants in Maden (ElazigTurkey). J Ethnopharmacol. 2011; 137: 469-486.

[93] Ozturk M, Uysal I, Gucel S, Altundag E, Dogan, Baslar S. Medicinal uses of natural dye-yielding plants in Turkey. RJTA. $2013 ; 17(2):$ 69-80.

[94] Sezik E, Yeşilada E, Honda G, Takaishi Y, Takeda Y, Tanaka T. Traditional medicine in Turkey IX: Folk medicine in north-west Anatolia. J Ethnopharmacol. 1999; 3: 195-210.

[95] Akbulut S, Bayramoglu MM. The trade and use of some medical and aromatic herbs in Turkey. Ethno Med. 2013; 7(2): 67-77.

[96] Altundag E, Ozturk M. Ethnomedicinal studies on the plant resources of east Anatolia, Turkey. Procedia Soc Behav Sci. 2011; 19 :756-777.

[97] Gürdal B, Kültür Ş. An ethnobotanical study of medicinal plants in Marmaris (Muğla, Turkey). J Ethnopharmacol. 2013; 1: 113-126.

[98] Sargin SA. Ethnobotanical survey of medicinal plants in Bozyazı district of Mersin, Turkey. J Ethnopharmacol. 2015; 173: 105-126.

[99] Sezik E, Yeşilada E, Honda G, Takaishi Y, Takeda Y, Tanaka T. Traditional medicine in Turkey X. Folk medicine in Central Anatolia. J Ethnopharmacol. 2001; 2-3: 95-115.

[100] Tetik F, Civelek S, Cakilcioglu U. Traditional uses of some medicinal plants in Malatya (Turkey). J Ethnopharmacol. 2013; 1: 331-346.

[101] Kaval I, Behçet L, Cakilcioglu U. Ethnobotanical study on medicinal plants in Geçitli and its surrounding (HakkariTurkey). J Ethnopharmacol. 2014; 1: 171-184.

[102] Mükemre M, Behçet L, Cakilcioglu U. Ethnobotanical study on medicinal plants in villages of Çatak (Van-Turkey). J Ethnopharmacol. 2015; 361-374.

[103] Polat R, Cakilcioglu U, Satıl F. Traditional uses of medicinal plants in Solhan (Bingöl - Turkey). J Ethnopharmacol. 2013; 148: 951-963.

[104] Güzel Y, Güzelşemme M, Miski M. Ethnobotany of medicinal plants used in Antakya: A multicultural district in Hatay Province of Turkey. J Ethnopharmacol. 2015; 174: 118 - 152.

[105] Hayta S, Polat R, Selvi S. Traditional uses of medicinal plants in Elazığ (Turkey). J Ethnopharmacol. 2014; 3: 613623.

[106] Polat R, Cakilcioglu U, Kaltalioğlu K, Ulusan MD, Türkmen Z. An ethnobotanical study on medicinal plants in Espiye and its surrounding (Giresun-Turkey). J Ethnopharmacol. 2015; 163: 1-11.

[107] Sargin SA, Selvi S, López V. Ethnomedicinal plants of Sarigöl district (Manisa). Turkey. J Ethnopharmacol. 2015; 171: $64-84$

[108] Fakir H, Korkmaz M, Güller B. Medicinal Plant Diversity of Western Mediterrenean Region in Turkey. JABS. 2009; 3(2): 30-40.

[109] Kilic O, Bagci E. An ethnobotanical survey of some medicinal plants in Keban (Elazı́g-Turkey). J Med Plants Res. 2013; 7(23): 1675-1684.

[110] Akgül G, Yılmaz N, Celep A, Celep F, Çakılcıŏlu U. Ethnobotanical purposes of plants sold by herbalists and folk bazaars in the center of Cappadocica (Nevşehir, Turkey). Indian J Tradit Knowle. 2016; 15(1): 103-108. 